\title{
Baicalin potentiates TRAIL-induced apoptosis through p38 MAPK activation and intracellular reactive oxygen species production
}

\author{
LEI ZHANG ${ }^{1}$, XIA WANG ${ }^{2}$, RUIXUE WANG $^{3}$, XUELIAN ZHENG $^{3}, \mathrm{NA} \mathrm{LI}^{3}$, \\ HUANNAN LI ${ }^{1}$, XIAOREN CAO ${ }^{1}$, BIN ZHOU $^{3}$, YONG LIN $^{3}$ and LAN YANG ${ }^{1}$
}

\begin{abstract}
${ }^{1}$ Department of Anatomy, Histology and Embryology, Chengdu University of Traditional Chinese Medicine, Chengdu, Sichuan 611137; ${ }^{2}$ Department of Immunology, West China School of Preclinical and Forensic Medicine; ${ }^{3}$ Laboratory of Molecular and Translational Medicine, Key Laboratory of Obstetric and Gynecologic and Pediatric Diseases and Birth Defects of Ministry of Education, Department of Gynecology and Obstetrics, West China Second University Hospital, Sichuan University, Chengdu, Sichuan 610041, P.R. China
\end{abstract}

Received December 23, 2016; Accepted August 11, 2017

DOI: $10.3892 / \mathrm{mmr} .2017 .7633$

\begin{abstract}
The combination of tumor necrosis factor-related apoptosis-inducing ligand (TRAIL) with other agents has been recognized as a promising strategy to overcome TRAIL resistance in cancer cells. Baicalin (5, 6-dihydroxy-7-o-glucuronide flavone) is a flavonoid from the root of the medicinal herb Scutellaria baicalensis Georgi, which has been reported to exert antioxidant, anti-inflammatory, antiviral and anticancer activities in vitro. However, the effect of baicalin on TRAIL-induced cytotoxicity has not been previously reported. In the present study, the effect of combining TRAIL and baicalin was investigated in non-small cell lung cancer cell lines. The results revealed that baicalin was able to sensitize A549 and H2009 cells to TRAIL-induced apoptosis. This was detected by the potentiation of poly-adenosine-5'-diphosphate-ribose polymerase cleavage and Annexin V-fluorescein isothiocyanate staining of cells co-treated with baicalin and TRAIL. In addition, p38 mitogen-activated protein kinase was activated in baicalin and TRAIL co-treated cancer cells, whereas the p38 inhibitor SB203580 effectively suppressed cell death within the co-treated cells. Butylated hydroxyanisole and $\mathrm{N}$-acetyl-cysteine, known reactive oxygen species (ROS) scavengers, significantly suppressed the potentiated cytotoxicity induced by baicalin and TRAIL co-treatment. The present study is the first, to the best of our knowledge, to demonstrate that baicalin enhances the anticancer activity of
\end{abstract}

Correspondence to: Dr Lan Yang, Department of Anatomy, Histology and Embryology, Chengdu University of Traditional Chinese Medicine, 1166 Liutai Avenue, Wenjiang, Chengdu, Sichuan 611137, P.R. China

E-mail: lanyang72@163.com

Key words: baicalin, lung cancer, apoptosis, p38 mitogen-activated protein kinase, reactive oxygen species
TRAIL via p38 activation and ROS accumulation, and may be exploited for anticancer therapy.

\section{Introduction}

Tumor necrosis factor (TNF)-related apoptosis-inducing ligand (TRAIL) is a potential anticancer agent. Numerous cancer cells have exhibited sensitivity to TRAIL-induced death, which has not been observed in normal cells. TRAIL binds to at least five receptors, including death receptors 4 and 5, which are functional TRAIL receptors. However, decoy receptors 1 and 2, and osteoprotegerin are decoy TRAIL receptors that inhibit the death-inducing activity of TRAIL (1). The application of TRAIL in cancer therapy is hindered due to TRAIL-associated resistance in cancer cells. The mechanisms of resistance may be associated with the dysfunction of TRAIL-induced signaling and/or high expression levels of anti-apoptotic molecules. TRAIL activates several cellular signaling pathways, including those involving mitogen-activated protein kinases (MAPKs), which result in apoptosis (2-4). Combining TRAIL with other agents to modulate these molecules or pathways may help to overcome TRAIL-associated resistance and therefore improve the therapeutic applications of TRAIL $(2,3)$.

Scutellaria baicalensis Georgi is a common Chinese medicinal herb, which contains various flavonoids, including baicalin, wogonin, wogonoside, oroxylin A and oroxylin A-7 (5). These flavonoids have various activities. Baicalin (5, 6-dihydroxy-7-o-glucuronide flavone) has been reported to possess a diverse range of pharmacological properties, including antioxidant, anti-inflammatory, antiviral and anticancer activities (6). Human leukemia, myeloma, breast, lung, bladder and lymphoma cancer cells were potently suppressed by this flavone as reported in previous studies (7-9). The molecular mechanisms underlying these effects are thought to involve alterations in oxidation/reduction status, cell cycle inhibition and the induction of apoptosis (6). However, the effect of baicalin on the anticancer activity of TRAIL has yet to be explored. 
In the present study, non-small cell lung cancer cell lines A549 and H2009 were treated with the combination of baicalin and TRAIL. Baicalin potently sensitized TRAIL-induced apoptosis of cancer cells via p38 MAPK activation and induction of intracellular reactive oxygen species (ROS) accumulation.

\section{Materials and methods}

Reagents. Glutathione S-transferase-TRAIL was purchased from SinoBio Biotech Ltd., (Shanghai, China). Baicalin was purchased from the National Institute of the Control Pharmaceutical and Biological Products (Beijing, China). Z-VAD-FMK was obtained from Calbiochem (EMD Millipore, Billerica, MA, USA). Butylated hydroxyanisole (BHA) and N-acetyl-L-cysteine (NAC) were purchased from Sigma-Aldrich (Merck KGaA,Darmstadt, Germany). 4-(4-fluorophenyl)-2-(4-methylsulfinylphenyl)-5-(4-pyridyl)-imidazole (SB203580) (cat. no. 152121-47-6) was obtained from Gene Operation (Ann Arbor, MI, USA). The JNK inhibitor SP600125 (cat. no. 129-56-6) was from EMD Millipore. The ERK inhibitor U0126 (cat. no. \#9903) was from Cell Signaling Technology Inc., (Danvers, MA, USA). CellROX Deep Red reagent was purchased from Thermo Fisher Scientific, Inc., (Waltham, MA, USA). The antibody poly (ADP-ribose) polymerase (PARP; cat. no. AP102) was obtained from Beyotime Institute of Biotechnology, Haimen, China). Anti-p38 MAPK (cat. no. \#9212) and anti-phosphorylated-p38 MAPK (Thr180/Tyr182) antibodies (cat. no. \#9211) were purchased from Cell Signaling Technology Inc., (Danvers, MA, USA). All of the above antibodies were diluted 1:1,000 in 5\% milk. Anti-GAPDH antibody (1:2,000; cat. no. 10494-1-AP) was obtained from Proteintech Group, Inc. (Chicago, IL, USA).

Cell culture. Two non-small cell lung cancer cell lines A549 and H2009 were from the American Type Culture Collection (ATCC, Manassas, VA, USA) and cultured in RPMI 1640 medium (cat. no. SH30809.01; Hyclone; GE Healthcare Life Sciences, Logan, UT, USA) supplemented with $10 \%$ fetal bovine serum (Hyclone; GE Healthcare Life Sciences), $1 \mathrm{mmol} / 1$ glutamate, $100 \mathrm{U} / \mathrm{ml}$ penicillin and $100 \mu \mathrm{g} / \mathrm{ml}$ streptomycin under standard incubator conditions at $37^{\circ} \mathrm{C}$ with $5 \% \mathrm{CO}_{2}$.

Cell death assay. Cells were seeded in a 96-well plate $24 \mathrm{~h}$ prior to treatment and were then treated with baicalin, TRAIL alone or in combination for $72 \mathrm{~h}$. Then culture medium from each well was collected and transferred to 96-well flat-bottomed plates. Lactate dehydrogenase (LDH) activity was determined by adding equal volumes of the reaction mixture to each well and incubating for $30 \mathrm{~min}$ at $22^{\circ} \mathrm{C}$. The absorbance of the samples was measured at $490 \mathrm{~nm}$ using a plate reader (Tecan Infinite F200). Cell death was detected quantitatively via lactate dehydrogenase (LDH) release using a cytotoxicity detection kit (Promega Corporation, Madison, WI, USA) as described previously (10). All experiments were repeated three to five times; the average is presented in each figure. Cell death was calculated using the formula: Cytotoxicity $(\%)=($ experimental value-spontaneous LDH release) / (maximum LDH release - spontaneous LDH release) x $100 \%$.
Analysis of apoptosis by flow cytometry. Apoptosis was detected by flow cytometry. A549 cells were seeded into a 6-well plate $24 \mathrm{~h}$ prior to treatment and were then treated with baicalin $(75 \mu \mathrm{M})$, TRAIL (30 $\mathrm{ng} / \mathrm{ml})$ alone or in combination for $48 \mathrm{~h}$. Subsequently, the cells were double stained with Annexin V-fluorescein isothiocyanate (V-FITC) and propidium iodide (PI) using an Annexin V-FITC Apoptosis Detection kit (Nanjing KeyGen Biotech Co., Ltd., Nanjing, China) according to the manufacturer's protocol. Early apoptosis is defined by Annexin $\mathrm{V}^{+} / \mathrm{PI}^{-}$staining (Q4) and late apoptosis is defined by Annexin $\mathrm{V}^{+} / \mathrm{PI}^{+}$staining (Q2,) as determined by fluorescence-activated cell sorting (Beckman Coulter, Inc., Brea, CA, USA).

Western blot analysis. A549 cells were seeded in a 6-well plate $24 \mathrm{~h}$ prior to treatment and were then treated with baicalin (75 $\mu \mathrm{M})$, TRAIL (30 ng/ml) alone or in combination for $72 \mathrm{~h}$. Cell extracts were then prepared by lysing cells in M2 buffer [20 mmol/1 Tris-HCL (pH 7.6), 0.5\% NP-40, $250 \mathrm{mmol} / 1$ $\mathrm{NaCl}, 3 \mathrm{mmol} / \mathrm{l}$ EDTA, $3 \mathrm{mmol} / \mathrm{l}$ EGTA, $2 \mathrm{mmol} / \mathrm{l}$ dithiothreitol, $0.5 \mathrm{mmol} / 1$ phenylmethylsulfonyl fluoride, $20 \mathrm{mmol} / \mathrm{l}$ $\beta$-glycerophosphate, $1 \mathrm{mmol} / 1$ sodium vanadate and $1 \mu \mathrm{g} / \mathrm{ml}$ leupeptin] and homogenizing them on ice. The extracts were subsequently incubated on ice for $30 \mathrm{~min}$. Protein concentration was determined using a Bradford protein assay kit (Beyotime Institute of Biotechnology, Haimen, China) according to the manufacturer's protocol. Protein samples $(50 \mu \mathrm{g})$ were separated by $10 \%$ SDS-PAGE. The proteins were transferred to a nitrocellulose filter membrane (Bio-Rad Laboratories, Inc., Hercules, CA, USA) following separation. The membrane was blocked with $5 \%$ bovine serum albumin (Sigma-Aldrich; Merck KGaA) in 1X Tris-buffered saline and $0.05 \%$ Tween-20 (TBST) for $1 \mathrm{~h}$ at room temperature with agitation. The membrane was then incubated with primary antibodies $(1: 1,000)$ at $4^{\circ} \mathrm{C}$ overnight, after which the membrane was washed three or five times with 1X TBST and was then incubated with the horse radish peroxidase-conjugated goat anti-rabbit immunoglobulin G (cat. no. ZB 2301; 1:2,000; OriGene Technologies, Inc., Rockville, MD, USA) for $1 \mathrm{~h}$ at room temperature with agitation. The proteins were visualized by enhanced chemiluminescence (Merck KGaA) using Bio-Rad Image Station (Bio-Rad Laboratories Inc.). Each experiment was repeated at least three times and representative results are shown in each figure.

Detection of ROS. Cells were cultured in 12-well plates overnight and subsequently treated with baicalin $(75 \mu \mathrm{M})$, TRAIL (30 ng/ml) alone or in combination for $3 \mathrm{~h}$. Cells were then stained for $30 \mathrm{~min}$ with $5 \mu \mathrm{M}$ CellROX Deep Red reagent (Thermo Fisher Scientific, Inc.), washed three times with precooled PBS, and analyzed using a Multiscan Spectrum plate reader (Thermo Fisher Scientific, Inc.). The wavelengths were set as follows: Excitation, $640 \mathrm{~nm}$; emission, $665 \mathrm{~nm}$. All the experiments were repeated at least three times, and representative results are shown in each figure $(11,12)$.

Statistical analysis. The presented data and results were confirmed in at least three independent experiments and were expressed as the mean \pm standard deviation. Student's t-test or one-way analysis of variance and a Tukey's post hoc 


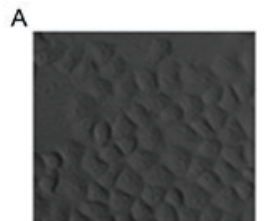

(-)

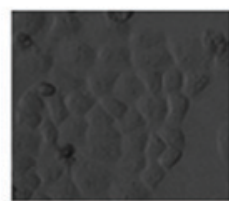

BAI

B
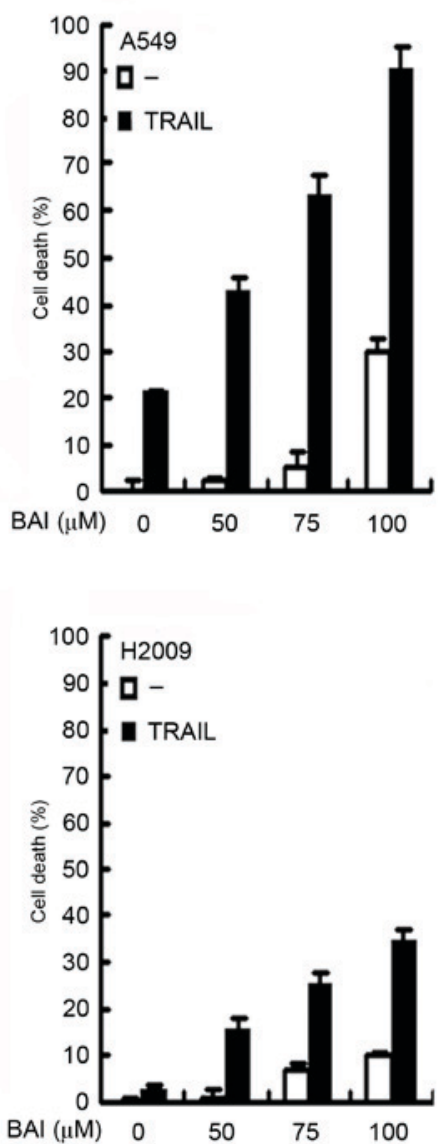

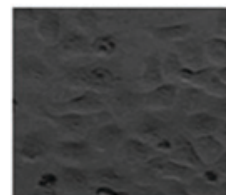

TRAIL

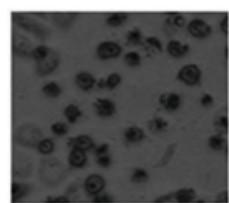

TRAIL+BAI

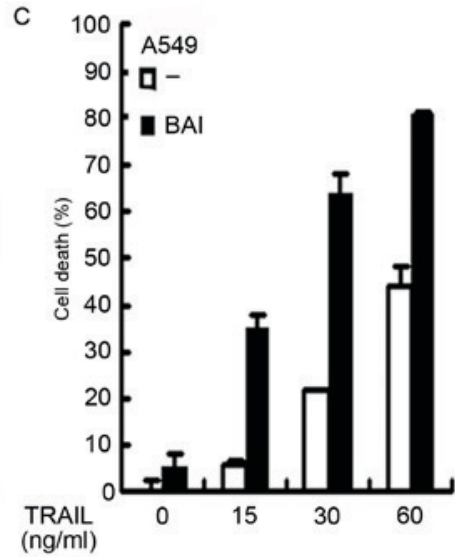

E

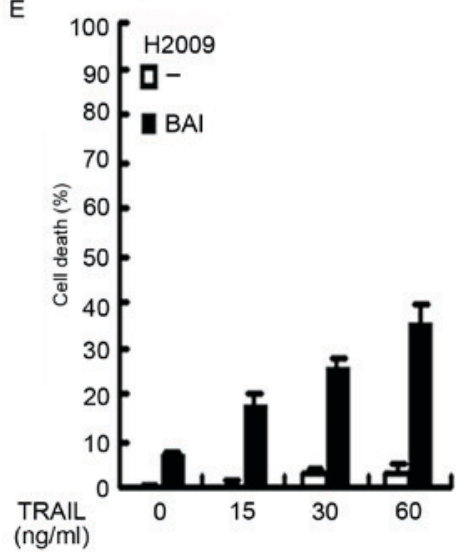

Figure 1. Baicalin sensitizes cancer cells to TRAIL-induced cytotoxicity. (A) A549 cells were treated with either $75 \mu \mathrm{M}$ baicalin, 30 ng/ml TRAIL or a combination for $72 \mathrm{~h}$. Cell death was observed microscopically (x40), representative images are shown. (B) A549 cells were treated with increasing concentrations of baicalin $(50-100 \mu \mathrm{M})$ or a fixed concentration of TRAIL $(30 \mathrm{ng} / \mathrm{ml})$ alone or in combination for $72 \mathrm{~h}$. (C) A549 cells were treated with a fixed concentration of baicalin $(75 \mu \mathrm{M})$ or increasing concentrations of TRAIL $(15-60 \mathrm{ng} / \mathrm{ml})$ alone or in combination for $72 \mathrm{~h}$. (D and E) H2009 cells were treated as described in (B and C), respectively. Cell death was measured using a lactate dehydrogenase release assay. Data are presented as the mean \pm standard deviation. TRAIL, tumor necrosis factor-related apoptosis-inducing ligand.

test were used for statistical analyses. The calculations were performed with SPSS 19.0 statistics software package (IBM Corp., Armonk, NY, USA). P<0.05 was considered to indicate a statistically significant difference.

\section{Results}

Baicalin enhances TRAIL-induced cell death in cancer cells. The present study investigated whether baicalin was able to enhance the anticancer activity of TRAIL in A549 cells. A549 cells were treated with $75 \mu \mathrm{M}$ baicalin, $30 \mathrm{ng} / \mathrm{ml}$ TRAIL alone, or a combination of both for $72 \mathrm{~h}$. Following treatment, cell death was observed microscopically. As demonstrated in the representative images (Fig. 1A), TRAIL or baicalin alone could induce only limited cell death, compared with the observed increase in cytotoxicity following co-treatment with baicalin and TRAIL. To quantitatively measure cell death, A549 cells were treated with increasing concentrations of baicalin (50-100 $\mu \mathrm{M}$ ) and a fixed concentration of TRAIL (30 ng/ml). Cell death was detected using a LDH release assay. While TRAIL alone caused $\sim 20 \%$ cell death, baicalin synergistically sensitized TRAIL-induced cell death in a dose-dependent manner (Fig. 1B). The synergism that killed $\sim 90 \%$ of cells was detected following treatment with TRAIL and the highest dose of baicalin $(100 \mu \mathrm{M})$, whereas this concentration of baicalin alone, was responsible for $\sim 30 \%$ of cell death. In addition, a similar dose-dependent synergistic effect of baicalin and TRAIL co-treatment was also observed with a fixed baicalin dose $(75 \mu \mathrm{M})$ and increasing concentrations of TRAIL (Fig. 1C). To exclude potential cell line-specific biases, the anticancer activity-associated sensitization of TRAIL was further validated in H2009 cells. As expected, a similar dose-dependent synergism with either a fixed concentration of TRAIL or baicalin was observed (Fig. 1D and E). 

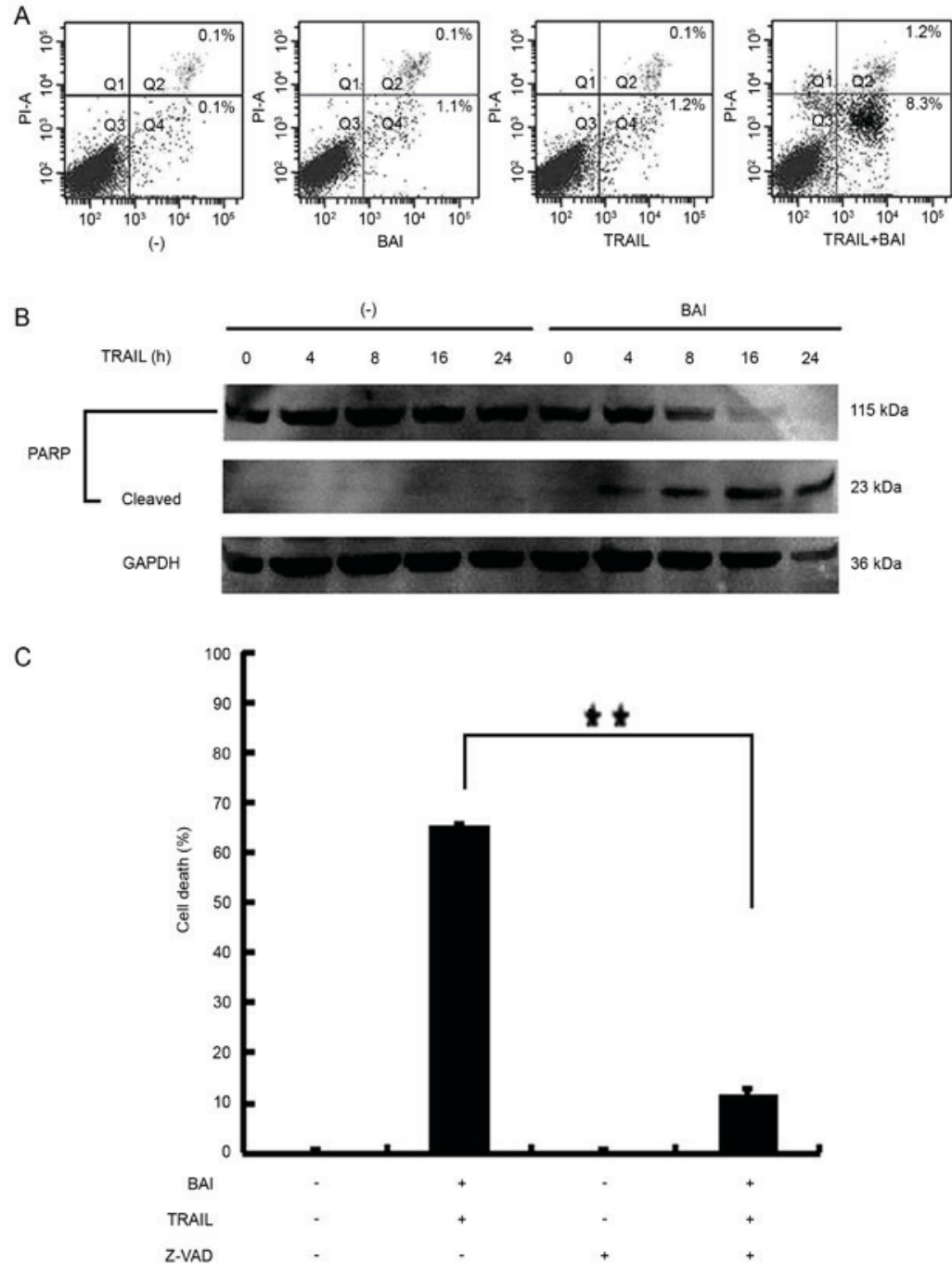

Figure 2. Baicalin enhances TRAIL-induced cancer cell apoptosis. (A) A549 cells were treated with baicalin (75 $\mu \mathrm{M})$, TRAIL (30 ng/ml) or both for $48 \mathrm{~h}$ and were then stained with Annexin V and PI followed by flow cytometric analysis. (B) A549 cells were treated with baicalin (75 $\mu \mathrm{M}$ ) for $1 \mathrm{~h}$ followed by TRAIL $(30 \mathrm{ng} / \mathrm{ml})$ treatment for various durations as indicated. PARP was detected by western blotting. GAPDH was detected as an input control. (C) A549 cells were pretreated with or without Z-VAD-FMK $(20 \mu \mathrm{M})$ for $1 \mathrm{~h}$, followed by baicalin $(75 \mu \mathrm{M})$ and TRAIL $(30 \mathrm{ng} / \mathrm{ml})$ for $72 \mathrm{~h}$. Cell death was measured using a lactate dehydrogenase release assay. Data are presented as the mean \pm standard deviation. ${ }^{\star \star} \mathrm{P}<0.01$. PARP, poly-adenosine-5'-diphosphate-ribose polymerase; PI, propidium iodide; TRAIL, tumor necrosis factor-related apoptosis-inducing ligand.

These results suggested that baicalin sensitized cancer cells to TRAIL-induced cytotoxicity.

Baicalin enhances TRAIL-induced apoptosis of cancer cells. Baicalin and TRAIL are able to induce apoptosis $(4,13,14)$. The present study investigated whether the increased cell death observed in lung cancer cells co-treated with baicalin and TRAIL was achieved through the potentiation of apoptosis. A549 cells were treated with baicalin, TRAIL alone or in combination. Subsequently, the cells were stained with Annexin V-FITC and PI; apoptosis was analyzed by flow cytometry. As shown in Fig. 2A, early apoptotic and late apoptotic cell populations were markedly increased with baicalin and TRAIL co-treatment. This finding indicated that the observed increased cell death stems from enhancing apoptosis. Western blot analysis also detected the activation of apoptosis. As depicted in Fig. 2B, the cleavage of the caspase substrate PARP was markedly increased in A549 cells treated with TRAIL and baicalin. As exhibited in Fig. 2C, A549 cells were pretreated with or without Z-VAD-FMK $(20 \mu \mathrm{M})$ for $1 \mathrm{~h}$, followed by baicalin $(75 \mu \mathrm{M})$ and TRAIL $(30 \mathrm{ng} / \mathrm{ml})$ for $72 \mathrm{~h}$. The enhancement of TRAIL-induced apoptosis by baicalin was significantly suppressed the synergistic cytotoxicity induced by co-treatment with TRAIL and baicalin $(\mathrm{P}<0.01)$.

p38 MAPK activation contributes to increased cytotoxicity induced by TRAIL and baicalin co-treatment. The apoptotic pathway is tightly regulated within the cell through multistep regulatory mechanisms. To investigate the mechanisms underlying increased cytotoxicity induced by baicalin and TRAIL co-treatment, several inhibitors were employed: a JNK inhibitor, SP600125; an ERK inhibitor, U0126; and a p38 inhibitor, SB203580. These inhibitors were used to block corresponding pathways in A549 cells treated with baicalin and TRAIL. The results of the present study revealed that only the p38 inhibitor SB203580 significantly inhibited the increased cytotoxicity induced by baicalin and TRAIL co-treatment; therefore, activation of the p 38 pathway may be 
A

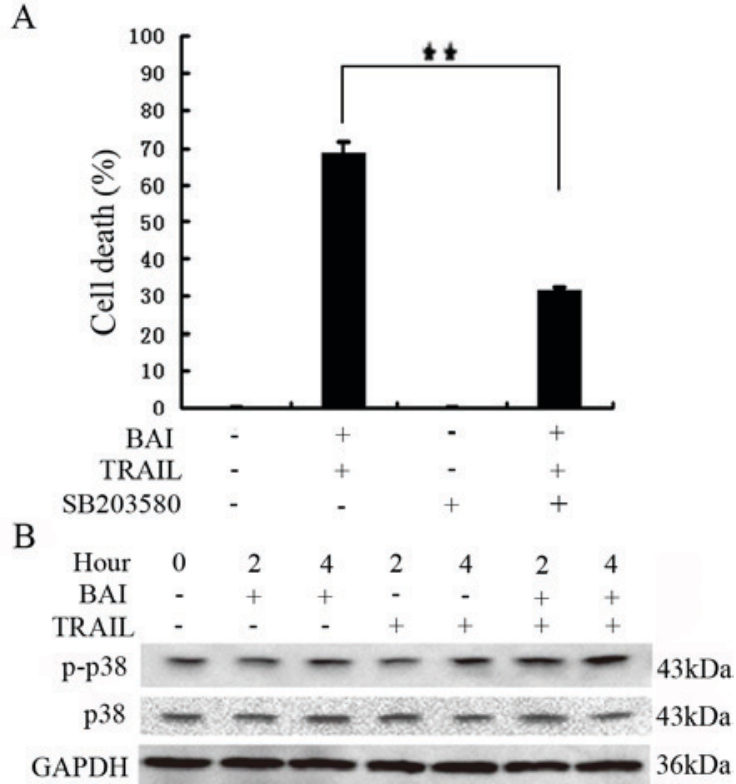

$\mathrm{C}$

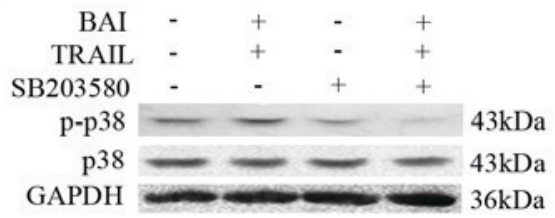

Figure 3. p38 inhibitor SB203580 suppresses synergistic cytotoxicity in baicalin and TRAIL co-treated A549 cells. Effects of baicalin, TRAIL and their combination on modulation of $\mathrm{p} 38$ mitogen-activated protein kinase signaling pathways in A549 cells. (A) A549 cells were pretreated with or without the p38 inhibitor SB203580 $(2 \mu \mathrm{M})$ for $1 \mathrm{~h}$, followed by baicalin $(75 \mu \mathrm{M})$ and TRAIL $(30 \mathrm{ng} / \mathrm{ml})$ treatment for $72 \mathrm{~h}$. Cell death was measured using a lactate dehydrogenase release assay. Data are presented as the mean \pm standard deviation. ${ }^{\star \star} \mathrm{P}<0.01$. (B) A549 cells were treated with baicalin $(75 \mu \mathrm{M})$ or TRAIL $(30 \mathrm{ng} / \mathrm{ml})$ individually or in combination for 2 and 4 h. p-p38 and p38 were detected by western blotting. GAPDH was detected as an input control. (C) A549 cells were pretreated with SB203580 $(2 \mu \mathrm{M})$ for $1 \mathrm{~h}$ followed by baicalin $(75 \mathrm{uM})$ and TRAIL $(30 \mathrm{ng} / \mathrm{ml})$ for $4 \mathrm{~h}$. p-p38 and p38 were detected by western blotting. GAPDH was detected as an input control. p-p38, phosphorylated-p38; TRAIL, tumor necrosis factor-related apoptosis-inducing ligand.

involved (Fig. 3A and data not shown). Consistently, whereas baicalin or TRAIL alone weakly activated p38, baicalin and TRAIL co-treatment markedly activated p38 (Fig. 3B), which could be suppressed by SB203580 (Fig. 3C). Altogether, these results suggested that the cytotoxic synergy of baicalin and TRAIL combination may be mediated by $\mathrm{p} 38$.

ROS accumulation contributes to the synergistic cytotoxicity induced by baicalin and TRAIL co-treatment. Since flavonoids affect cellular ROS status and excessive ROS are cytotoxic to cells, the role of ROS in baicalin and TRAIL-induced synergistic cytotoxicity was examined. ROS scavengers BHA and NAC were applied to A549 cells as a pretreatment prior to co-treatment with baicalin and TRAIL. The result demonstrated that BHA and NAC effectively suppressed the synergistic cytotoxicity in A549 cells co-treated with baicalin and TRAIL (Fig. 4A). The combination of baicalin and TRAIL induced significant
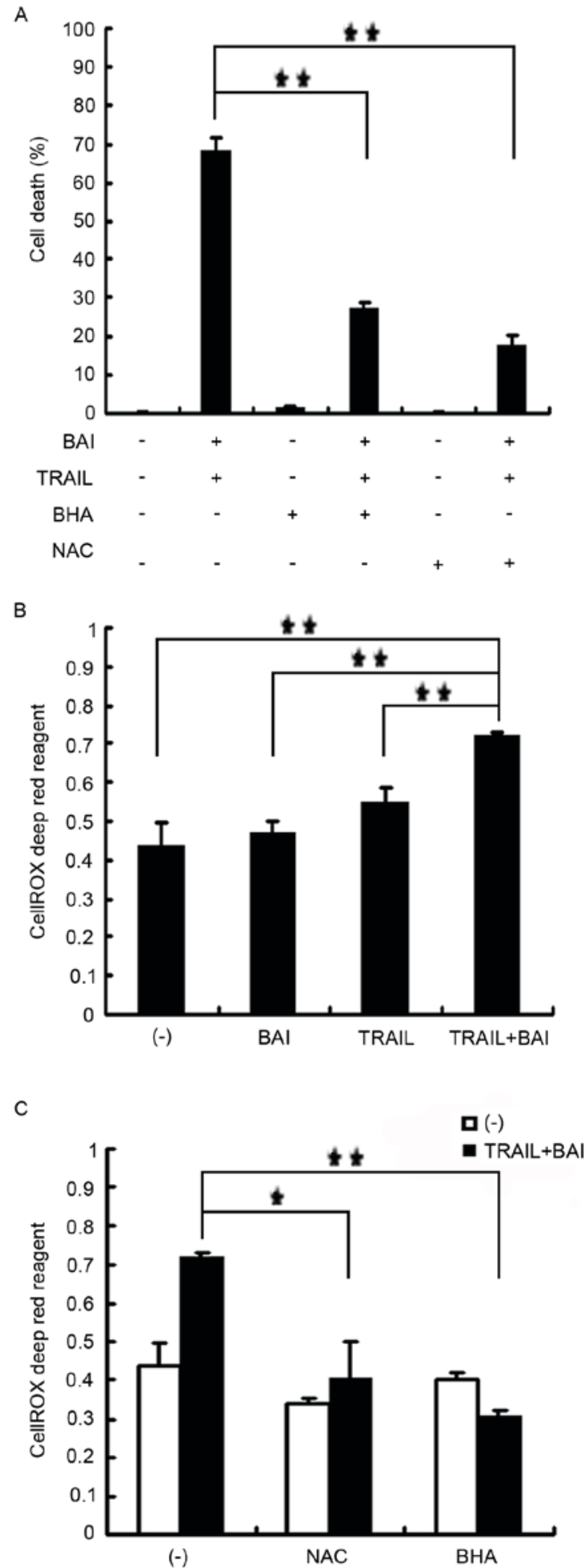

Figure 4. Intracellular ROS accumulation contributes to the synergistic cytotoxicity induced by baicalin plus TRAIL in A549 cells. (A) A549 cells were pretreated with or without BHA $(80 \mu \mathrm{M})$ or NAC $(800 \mu \mathrm{M})$ for $1 \mathrm{~h}$, followed by baicalin $(75 \mu \mathrm{M})$ and TRAIL $(30 \mathrm{ng} / \mathrm{ml})$ treatment for $72 \mathrm{~h}$. Cell death was measured using a lactate dehydrogenase release assay. (B) A549 cells were treated with baicalin $(75 \mu \mathrm{M})$ or TRAIL $(30 \mathrm{ng} / \mathrm{ml})$ or in combination for $3 \mathrm{~h}$. Cells were stained with CellROX Deep Red reagent $30 \mathrm{~min}$ prior to collecting cells and were subsequently analyzed. (C) A549 cells were pretreated with or without BHA $(80 \mu \mathrm{M})$ or NAC $(800 \mu \mathrm{M})$ for $1 \mathrm{~h}$ and were then co-treated with baicalin $(75 \mu \mathrm{M})$ and TRAIL $(30 \mathrm{ng} / \mathrm{ml})$ for a further $3 \mathrm{~h}$. Cells were stained with CellROX Deep Red reagent $30 \mathrm{~min}$ prior to collecting cells and were then analyzed. Data are presented as the mean \pm standard deviation. ${ }^{\star} \mathrm{P}<0.05,{ }^{\star}{ }^{\star} \mathrm{P}<0.01$. BHA, butylated hydroxyanisole; NAC, N-acetyl-L-cysteine; TRAIL, tumor necrosis factor-related apoptosis-inducing ligand. 
ROS accumulation within the A549 cells, as detected by the CellROX Deep Red reagent (Fig. 4B); however, BHA and NAC effectively suppressed ROS accumulation (Fig. 4C). However, the ROS scavengers had little effect on p38 activation (data not shown), suggesting that p38 and ROS may be independently involved in cell death caused by baicalin and TRAIL co-treatment.

\section{Discussion}

TRAIL is considered the most promising therapy within the TNF family for anticancer treatment, as it selectively induces death within tumor cells but not in normal cells (15). The combination of TRAIL with conventional chemotherapy drugs or radiotherapy can enhance cytotoxicity toward cancer cells. Previous studies have demonstrated that alone baicalin has numerous anticancer activities (7-9).

In the present study, whether baicalin could effectively potentiate TRAIL-induced cytotoxicity in cancer cells was examined. Treating cancer cell lines with baicalin and TRAIL resulted in synergistic cytotoxicity in a dose-dependent manner. Furthermore, the synergistic cytotoxicity induced by baicalin and TRAIL was revealed to be due to increased apoptosis, as determined by Annexin V staining, detection of PARP cleavage and cell death inhibition by the pan-caspase inhibitor Z-VAD-FMK.

The involvement of p38 and ROS in baicalin and TRAIL-induced cell death was subsequently investigated. It is known that MAPKs, including p38, serve an important role in drug response during cancer chemotherapy (16-18). It was demonstrated in the present study that p38 MAPK was significantly activated in TRAIL and baicalin co-treated cancer cells; however, the p38 inhibitor SB203580 was able to suppress increased cell death. ROS are important modulators of cellular signaling for apoptosis. Baicalin and TRAIL co-treatment significantly induced ROS accumulation, whereas ROS scavengers BHA and NAC markedly suppressed the potentiated cytotoxicity caused by baicalin and TRAIL co-treatment. Consistently, our previous study demonstrated that wogonin, another important component of S. baicalensis Georgi, activates ROS to promote TRAIL-induced apoptosis (13). It is also known that the activation of p38 is often mediated by ROS (19-22). However, the results of the present study revealed that baicalin and TRAIL-induced activation of $\mathrm{p} 38$ is independent of ROS.

Therefore, the present study demonstrated that baicalin could be used as a potential TRAIL sensitizer for cancer therapy by regulating ROS degradation and the p38 MAPK signaling pathway. Further studies are required to elucidate the mechanisms of the ROS and p38 MAPK pathway interaction in baicalin and TRAIL-induced cell death. The results of the present study add to the knowledge of the anticancer value of these naturally occurring compounds and indicate novel therapeutic targets for lung cancers.

\section{Acknowledgements}

The present study was supported by grants from the Science \& Technology Department of Sichuan Province (grant no. 2014JY0035) and the Chengdu University of TCM (grant nos. ZRYY201209 and ZRYY201310).

\section{References}

1. Emery JG, McDonnellP, Burke MB, Deen KC, Lyn S, Silverman C, Dul E, Appelbaum ER, Eichman C, DiPrinzio R, et al: Osteoprotegerin is a receptor for the cytotoxic ligand TRAIL. J Biol Chem 273: 14363-14367, 1998.

2. Grotzer MA, Eggert A, Zuzak TJ, Janss AJ, Marwaha S, Wiewrodt BR, Ikegaki N, Brodeur GM and Phillips PC: Resistance to TRAIL-induced apoptosis in primitive neuroectodermal brain tumor cells correlates with a loss of capase- 8 expression. Oncogene 19: 4604-4610, 2000.

3. Wang X, Chen W, Zeng W, Bai L, Tesfaigzi Y, Belinsky SA and Lin Y: Akt-mediated eminent expression of c-FLIP and Mcl-1 confers acquired resistance to TRAIL-induced cytotoxicity to lung cancer cells. Mol Cancer Ther 7: 1156-1163, 2008.

4. Yang J, Li G and Zhang K: Pro-survival effects by NF-кB, Akt and $\operatorname{ERK}(1 / 2)$ and anti-apoptosis actions by Six1 disrupt apoptotic functions of TRAIL-Dr4/5 pathway in ovarian cancer. Biomed Pharmacother 84: 1078-1087, 2016.

5. Li K and Sheu S: Determination of flavonoids and alkaloids in the scute-coptis herb couple by capillary electrophoresis. Anal Chim Acta 313: 113-120, 1995.

6. Srinivas NR: Baicalin, an emerging multi-therapeutic agent: Pharmacodynamics, pharmacokinetics, and considerations from drug development perspectives. Xenobiotica 40: 357-367, 2010.

7. Lee D, Ko WK, Hwang DS, Heo DN, Lee SJ, Heo M, Lee KS, Ahn JY, Jo J and Kwon IK: Use of baicalin-conjugated gold nanoparticles for apoptosis induction of breast cancer cells. Nanoscale Res Lett 11: 381, 2016.

8. Orzechowska B, Chaber R, Wiśniewska A, Pajtasz-Piasecka E, Jatczak B, Siemieniec I, Gulanowski B, Chybicka A and Błach-Olszewska Z: Baicalin from the extract of Scutellaria baicalensis affects the innate immunity and apoptosis in leukocytes of children with acute lymphocytic leukemia. Int Immunopharmacol 23: 558-567, 2014

9. Huang Y, Hu J, Zheng J, Li J, Wei T, Zheng Z and Chen Y: Down-regulation of the PI3K/Akt signaling pathway and induction of apoptosis in CA46 Burkitt lymphoma cells by baicalin. J Exp Clin Cancer Res 31: 48, 2012.

10. Wang X, Ju W, Renouard J, Aden J, Belinsky SA and Lin Y: 17-allylamino-17-demethoxygeldanamycin synergistically protentiates tumor necrosis factor-induced lung cancer cell death by blocking the nuclear factor-kappaB pathway. Cancer Res 66: 1089-1095, 2006.

11. Ju W, Wang X, Shi H, Chen W, Belinsky SA and Lin Y: A critical role of luteolin-induced reactive oxygen species in blockage of tumor necrosis factor-activated nuclear factor-kappaB pathway and sensitization of apoptosis in lung cancer cells. Mol Pharmacol 71: 1381-1388, 2007.

12. Saidani C, Hammoudi-Triki D, Laraba-Djebari F and Taub M: In vitro studies with renal proximal tubule cells show direct cytotoxicity of Androctonus australis hector scorpion venom triggered by oxidative stress, caspase activation and apoptosis. Toxicon 120: 29-37, 2016.

13. Yang L, Wang Q, Li D, Zhou Y, Zheng X, Yan J, Zhang L, Lin $Y$ and Wang $X$ : Wogonin enhances antitumor activity of tumor necrosis factor-related apoptosis-inducing ligand in vivo through ROS-mediated downregulation of cFLIPL and IAP proteins. Apoptosis 18: 618-626, 2013.

14. Refaat A, Abdelhamed S, Saiki I and Sakurai H: Inhibition of p38 mitogen-activated protein kinase potentiated the apoptotic effect of berberine/tumor necrosis factor-related apoptosis-inducing ligand combination therapy. Oncol Lett 10: 1907-1911, 2015.

15. Walczak H, Miller RE, Arial K, Gliniak B, Griffith TS, Kubin M, Chin W, Jones J, Woodward A, Le T, et al: Tumoricidal activity of tumor necrosis factor-related apoptosis-inducing ligand in vivo. Nat Med 5: 157-163, 1999.

16. Galan-Moya EM, de la Cruz-Morcillo Ma, Llanos Valero M, Callejas-Valera JL, Melgar-Rojas P, Hernadez Losa J, Salcedo M, Fernández-Aramburo A, Ramon y Cajal S and Sánchez-Prieto R: Balance between MKK6 and MKK3 mediates p38 MAPK associated resistance to cisplatin in NSCLC. PLoS One 6: e28406, 2011.

17. Xie Y, Peng Z, Shi M, Ji M, Guo H and Shi H: Metformin combined with p38 MAPK inhibitor improves cisplatin sensitivity in cisplatin-resistant ovarian cancer. Mol Med Rep 10: 2346-2350, 2014. 
18. Pal HC, Baxter RD, Hunt KM, Agarwal J,Elmets CA, Athar M and Afaq F: Fisetin, a phytochemical, potentiates sorafenib-induced apoptosis and abrogates tumor growth in athymic nude mice implanted with BRAF-mutated melanoma cells. Oncotarget 6: 28296-28311, 2015.

19. Pereira L, Igea A, Canovas B, Dolado L and Nebreda AR: Inhibition of p38 MAPK sensitizes tumour cells to cisplatin-induced apoptosis mediated by reactive oxygen species and JNK. EMBO Mol Med 5: 1759-1774, 2013.

20. Shi Y, Nikulenkov F, Zawacka-Pankau J, Li H, Gabdoulline R, $\mathrm{Xu}$ J, Eriksson S, Hedström E, Issaeva N, Kel A, et al: ROS-dependent activation of JNK converts p53 into an efficient inhibitor of oncogenes leading to robust apoptosis. Cell Death Differ 21: 612-623, 2014
21. Zou J, Zou P, Lou Y, Xiao Y, Wang J and Liu L: The cross-talk between ROS and p38MAPK $\alpha$ in the ex vivo expanded human umbilical cord blood CD133(+) cells. J Huazhong Univ Sci Technolog Med Sci 31: 591-595, 2011.

22. Chen Z, Jiang H, Wan Y, Bi C and Yuan Y: H(2)O(2)-induced secretion of tumor necrosis factor- $\alpha$ evokes apoptosis of cardiac myocytes through reactive oxygen species-dependent activation of p38 MAPK. Cytotechnology 64: 65-73, 2012. 